\title{
ACUTE KIDNEY INJURY
}

\section{Renal hazards of anticoagulant therapy}

The anticoagulant dabigatran induces glomerular haemorrhage and renal tubular obstruction, which resembles warfarin-related nephropathy (WRN), say researchers at the Ohio State University, USA. "Our most significant finding is that glomerular haemorrhage is not related to warfarin only, but rather to anticoagulation itself," states lead investigator Sergey Brodsky.

Anticoagulant therapy with warfarin is widely recognized to increase the risk of acute kidney injury; however, little is known about the renal risks associated with other classes of anticoagulants. The researchers report that elevations in serum creatinine and haematuria were induced by treatment with the anticoagulant dabigatran in both control and 5/6 nephrectomized rats (a model of chronic kidney disease). In comparison with warfarin, which causes kidney injury only in 5/6 nephrectomized rats, these observations suggest that dabigatran might have direct nephrotoxic effects.
Dabigatran increased renal tubular obstruction, due to red blood cell casts, in 5/6 nephrectomized rats, but only occasionally in control rats. Similar, but weaker, effects were seen with the antagonist SCH79797, which inhibits the thrombin receptor protease-activated receptor 1 (PAR-1), suggesting that the effect of dabigatran on the kidney is mediated in part via PAR-1.

This study confirms the risk of acute kidney injury from anticoagulants and indicates that WRN might be part of a wider syndrome, which the investigators term anticoagulant-related nephropathy. "All patients on anticoagulation should be monitored for renal function changes," recommends Brodsky.

David Holmes

Original article Ryan, M. et al. Warfarin-related nephropathy is the tip of the iceberg: direct thrombin inhibitor dabigatran induces glomerular hemorrhage with acute kidney injury in rats. Nephrol. Dial. Transplant. doi:10.1093/ndt/gft380 